\title{
FRINGE FAMILIES IN STABLE HOMOTOPY
}

\author{
BY
}

RAPHAEL S. ZAHLER

\begin{abstract}
It is shown how to detect and construct elements in the stable homotopy groups of spheres corresponding to the $\epsilon_{i}$ family of Toda. The only tools used are Brown-Peterson cohomology and the Adams spectral sequence.
\end{abstract}

Recent work on the $p$-primary stable homotopy groups of spheres has shown that it is possible to give a unified interpretation of the three infinite families $\alpha_{t}, \beta_{t}, \gamma_{t}$ of indecomposable elements ( $p$ is a prime at least 5). The method involves constructing the stable maps by factoring through " $V(n)$-spaces"spaces whose Brown-Peterson cohomology has a simple structure. This cohomology theory also provides the tool for "detecting" the resulting stable maps (showing that they are nontrivial).

Since Toda has also discovered the indecomposable stable-stem elements $\epsilon_{1}, \ldots, \epsilon_{p-1}, \epsilon^{\prime}$, and $\phi[16]$, we tried to fit them into this general scheme as well. It turned out that the $\epsilon_{i}$ were just the beginning of an infinite family. This new family, whose elements trail like a fringe effect behind each $\beta_{t}$, has a pleasingly rhythmic structure on the algebraic level (the $E_{2}$-term of the $B P$ Adams spectral sequence), and at least part of this structure carries over to the stable stems themselves:

THEOREM 1. (a) (DETECTION). There exist nonzero elements e(i, ap $)$ of order $p$ in the $E_{2}$-term of the BP Adams spectral sequence [19] for each prime $p \geqslant 3$, and $f \geqslant 1,(a, p)=1,0 \leqslant i \leqslant p^{f}-1$. The bidegree of $e\left(i, a p^{f}\right)$ is $(2$, $\left.\left(a p^{f+1}+(a-1) p^{f}+i\right) q\right)$, where $q=2 p-2$.

(b) (Construction). For $p \geqslant 5$, and $1 \leqslant p^{f}-i \leqslant p-1$ at least, $e\left(i, a p^{f}\right)$ survives to $E_{\infty}$, representing a nontrivial element $\epsilon(i, a p f)$ in the stable homotopy of spheres, such that $1, p \geqslant 5$.

(c) $\epsilon\left(p^{f}-1, a p^{f}\right)=\beta_{a p}, \epsilon(i, p)=\epsilon_{i}$ up to units in $Z / p$, for $1 \leqslant i \leqslant p-$

Parts (a), (b), and (c) of the theorem are proved in $\S \S 1,2$, and 3, respectively.

Thus on the $E_{2}$-level the number of $e$ 's decorating each $\beta_{t}$ is one less than

Received by the editors June $26,1975$.

AMS (MOS) subject classifications (1970). Primary 55G25. 
the power of $p$ in $t$. In fact, we can think of $\epsilon\left(i, a p^{f}\right)$ as $\left(1 / v_{1}^{p^{f}-1-i}\right) \beta a p^{f}$, where $v_{1}$ is an element of bidegree $(0, q)$; thus we can say that $\beta_{a p} f$ is divisible by $v_{1}^{p^{f}-1}$. This is reminiscent of the Bernouilli-number description of the image of $J: \alpha_{a p} f$ is divisible by $p^{f}$. It is possible to write down similar fringe elements on the $p$ th level of $E_{2}$ for all $n$, but we cannot say whether any of them are nonzero.

Recently Miller and Wilson have definitively improved the divisibility results of (a) [9].

After we derived these facts in April 1974, we learned that Larry Smith had obtained Theorem 1 (b) and (c) by different methods [14]. S. Oka has also proved (c) and a somewhat stronger version of (b) [11]. While our proof probably came somewhat later than these, we feel that it has the advantage of assuming no prior information about the stable stems; it is also shorter.

We do not know whether (b) and (c) carry over to the $p=3$ case. Tangora's recent computations [15] may be helpful here.

The organization of the paper is simple: first detection, then construction. The latter depends on a third section in which we simplify greatly some of the calculational techniques in [19], linking $\operatorname{Ext}_{B P^{*}(B P)}$ to Toda's computation of Ext $_{p}$, where $P$ is the algebra of Steenrod $p$ th powers. Using this, we recover a theorem of Miller on edge periodicity in the BP Adams spectral sequence.

We would like to thank Haynes Miller, Larry Smith, Steve Wilson, and Dave Johnson for their help. J. F. Adams' Notre Dame lecture of 1973 was an important stimulus. The author was partially supported by the N.S.F.

1. Invariant ideals and twisted short exact sequences. An ideal $I \subset B P^{*}$ $\cong Z_{(p)}\left[v_{1}, v_{2}, \ldots\right]$ (notation as in [19]) is called invariant if for all $x \in I$ and $r \in B P^{*}(B P)$ we have $r x \in I$. For example, the annihilator ideal of any primitive element of any $B P^{*}(X)$ is always invariant [21, Corollary 3]. Landweber has shown that the only invariant prime ideals of $B P^{*}$ are those of the form $\left(p, v_{1}, \ldots, v_{n}\right), n \geqslant 0[6]$. But no simple description of invariant ideals in general is known. It is easy to see that $(p, x)$ is invariant if and only if $x=$ $v_{1}^{r}, r \geqslant 0$. Going a step further, we can prove

LEMMA 2. Let $(a, p)=1, f \geqslant 0$; then $\left(p, v_{1}^{r}, v_{2}^{a p^{f}}\right)$ is invariant if and only if $r \leqslant p^{f}$.

Proof. Let $r=\Sigma_{K} r_{K}$, where the sum runs over all exponent sequences $K=\left(k_{1}, k_{2}, \ldots\right)\left[19\right.$, p. 484]. Then $r(x y)=r(x) r(y)$ for $x, y \in B P^{*}$. But we know $r\left(v_{2}\right)=v_{2}-v_{1}^{p}+v_{1} \bmod p[21,(3)]$. Hence 


$$
\begin{aligned}
r\left(v_{2}^{a p^{f}}\right) & =\left(r v_{2}\right)^{a p^{f}}=\left[\left(v_{2}-v_{1}^{p}+v_{1}\right)^{p^{f}}\right]^{a} \bmod p \\
& =\left(v_{2}^{p^{f}}-v_{1}^{p^{f+1}}+v_{1}^{p}\right)^{a} \bmod p \\
& =v_{2}^{a p^{f}}+a v_{1}^{p^{f}} v_{2}^{(a-1) p^{f}}+\text { other terms in }\left(p, v_{1}^{p^{f}}\right) .
\end{aligned}
$$

The lemma is now obvious.

We now introduce the Ext groups, where the ground ring $A=B P *(B P)$ is always understood unless otherwise specified. By the lemma, if $N=$ $a p^{f}(p+1) q$ the group

$$
\operatorname{Ext}^{0, N}\left(B P^{*}, B P^{*} /\left(p, v_{1}^{\eta}\right)\right)=\operatorname{Hom}_{A}^{N}\left(B P^{*}, B P^{*} /\left(p, v_{1}^{r}\right)\right)
$$

contains the $B P^{*}$-linear homomorphism which sends $1 \in B P *$ to $v_{2}^{a p^{f}}$, if $r<p^{f}$. Let us abuse notation slightly and call this homomorphism $v_{2}^{a p^{f}}$. Using the short exact sequences

$$
\begin{aligned}
& E_{1}: 0 \rightarrow B P^{*} \stackrel{p}{\longrightarrow} B P^{*} \stackrel{j_{1}}{\longrightarrow} B P^{*} /(p) \rightarrow 0, \\
& E_{2}: 0 \rightarrow S^{r q} B P^{*} /(p) \stackrel{v_{1}^{r}}{\longrightarrow} B P^{*} /(p) \stackrel{j_{2}}{\longrightarrow} B P^{*} /\left(p, v_{1}\right) \rightarrow 0,
\end{aligned}
$$

we can set up the diagram

$$
\underset{\operatorname{Ext}^{0}{ }^{0, N}\left(B P^{*}, B P^{*} /(p)\right) \stackrel{j_{2}}{\longrightarrow} \operatorname{Ext}^{0, N}\left(B P^{*}, B P^{*} /\left(p, v_{1}\right)\right)}{\mid\left(E_{2}\right)}
$$

where $r=p^{f}-i$. We then define

$$
e\left(i, a p^{f}\right)=E_{1} * E_{2} * v_{2}^{a p^{f}} \text {, where } i=p^{f}-r \text {. }
$$

It is sometimes helpful to think of $E_{2} * v_{2}^{a p^{f}}$ as the short exact sequence whose twisting cochain is given by the formula $r_{E} \lambda_{1}=\left(1 / v_{1}\right) r_{E} v_{2}^{a p} \lambda_{0} \bmod p$ $[5,2.10]$.

Proof of Theorem 1(a). To prove that $e\left(i, a p^{f}\right)$ is nonzero it suffices to show that $j_{2}$ is zero, and that $E_{2} * v_{2}^{a p^{f}}$ is not in the image of $j_{1}$. For this we may apply the proof of $[5,2.11]$ verbatim. It is much easier, however, to to invoke the complete calculation of $\operatorname{Ext}^{0}\left(B P^{*}, B P^{*} /(p)\right)[6]$ and of $\operatorname{Ext}^{1}\left(B P^{*}, B P^{*}\right)[10]$ to complete the proof.

Notes. (1) A generalization of this argument is one of the key results of [4] 
(2) Theorem 1(a) includes and supersedes 5.8 and 5.11 of [19].

We can now use Theorem 1(a) to detect the $\epsilon$ 's, once we explain the basic idea of the program for their construction. Let $V(0)=S^{0} \cup_{p} e^{1}$ in stable notation, with natural maps $S^{0} \stackrel{i}{\rightarrow} V(0) \stackrel{\pi}{\rightarrow} S^{1}$ (for full information on $V(n)$ spaces, consult [18]). Let $\alpha: S^{q} V(0) \rightarrow V(0)$ be the stable map with $\pi \alpha i=\alpha_{1}$, the element of Hopf invariant one $\bmod p$ [1]. If $W=W_{r}$ is the mapping cone of $\alpha^{r}$, then $B P^{*}(W)=B P^{*}\left(C \alpha^{r}\right)=S^{r q+2} B P^{*} /\left(p, v_{1}^{r}\right)$. Following the pattern of [12] , [20], and [18], then, we would like to find maps $\epsilon_{f}: S^{N} W \rightarrow W$ for suitable $N$, with $B P^{*}\left(\epsilon_{f}\right)$ being multiplication by $v_{2}^{p}$. We could then generate interesting stable-stem families in the usual way. If such an $\epsilon_{f}$ existed, however, the annihilator ideal of the canonical generator of $B P^{*}\left(C \epsilon_{f}\right)$ would be $\left(p, v_{1}^{r}\right.$, $\left.v_{2}^{p^{f}}\right)$. Hence by Lemma 2 a necessary condition for the existence of $\epsilon_{f}$ is $r \leqslant$ $p^{f}$.

We will construct maps $\epsilon_{f}$ for $p \geqslant 5$ and $r \leqslant p-1$. For each such value of $r$ we will first find a map $\bar{\epsilon}: S^{N} W \rightarrow S^{0}$ such that $B P^{*}(\bar{\epsilon}): B P^{*} \rightarrow S^{N} B P^{*} W$ realizes multiplication by $v_{2}^{p}$, using a spectral sequence comparison argument. We will then use a multiplication $W \wedge W \rightarrow W$ to extend $\bar{\epsilon}$ to a map $\epsilon: S^{N} W$ $\rightarrow W$ inducing multiplication by $v_{2}^{p}$. Then we will define $\epsilon\left(t, a p^{f}\right)$ as the composite

$$
\left.S^{a p^{f-1}} N \stackrel{i}{\longrightarrow} S^{a p^{f-1}} N V(0) \stackrel{i_{r}}{\longrightarrow} S^{a p^{f-1} N} W \stackrel{\epsilon}{\longrightarrow} S^{(a p} p^{f-1}-1\right) N W
$$

$$
\stackrel{\epsilon}{\longrightarrow} \cdots \stackrel{\epsilon}{\longrightarrow} W \stackrel{\pi_{r}}{\longrightarrow} S^{r q+2},
$$

where $t=p^{f}-r$ and $i, i_{r}$ are the usual inclusions, and $\pi_{r}$ is the collapse onto the top cell.

Since for $r=1$ we have $W=V(1)$, we can take $\epsilon=\beta: S^{(p+1) q} V(1) \rightarrow$ $V(1)$, the map discovered by Smith [12]. Thus

$$
\epsilon\left(p^{f}-1, a p^{f}\right)=\beta_{a p^{f}}
$$

Another corollary is a realization theorem for some of the ideals in Lemma 2.

Proposition 5. For $p \geqslant 5, r \leqslant p-1$, and $j \geqslant 1$, there is a complex $X$ with $B P_{*}(X) \subseteq B P_{*} /\left(p, v_{1}^{r}, v_{2}^{j p}\right)$.

Proof. Let $X$ be the mapping cone of the $j$-fold iteration of $\epsilon$.

It is not known how many maps $\epsilon_{f}$ can be constructed for $r \geqslant p$. We can show, however, that whenever a map $\epsilon_{f}$ exists, the corresponding maps $\epsilon\left(i, a p^{f+j}\right)$ in stable homotopy obtained by inclusion of the bottom cell of $W$, iteration of $\epsilon_{f}$, and collapse onto the top cell, are essential. We do this by showing 
that the secondary $B P$ invariant for $\epsilon\left(i, a p^{f}\right)$ is $e\left(i, a p^{f}\right)$, which is nontrivial.

Given an element $\zeta \in \pi_{k}^{S}$ of order $p$, form an extension $\hat{\zeta}: S^{k} V(0) \rightarrow S^{0}$. When we apply $B P^{*}$ to the cofiber sequence of $\hat{\xi}$, we obtain a short exact sequence

$$
E: \quad 0 \rightarrow S^{k+2} B P^{*} /(p) \rightarrow B P^{*}(C \hat{\zeta}) \rightarrow B P^{*} \rightarrow 0 .
$$

We think of $E$ as an element of $\operatorname{Ext}_{A}^{1}\left(B P^{*}, B P^{*} /(p)\right)$. Although $E$ depends on the choice of extension $\hat{\zeta}$, the class of $E$ in $\operatorname{Ext}^{1}\left(B P^{*}, B P^{*} /(p)\right) / \pi^{*} \operatorname{Ext}^{1}\left(B P^{*}, B P^{*}\right)$ is a well-defined invariant of $\zeta$. Since $j_{1}$ in diagram (3) corresponds to $\pi^{*}$, the monomorphism

$$
E_{1 *}: \frac{\operatorname{Ext}^{1}\left(B P^{*}, B P^{*} /(p)\right)}{\pi^{*} \operatorname{Ext}^{1}\left(B P^{*}, B P^{*}\right)} \hookrightarrow \operatorname{Ext}^{2}\left(B P^{*}, B P^{*}\right)
$$

gives us an invariant in $\mathrm{Ext}^{2}$.

Proposition 6. This $\mathrm{Ext}^{2}$-invariant of $\epsilon\left(i, a p^{f}\right)$ is $e\left(i, a p^{f}\right)$.

Proof. Since $e\left(i, a p^{f}\right)=E_{1} * E_{2} * v_{2}^{a p^{f}}$, it suffices to show that the class of the sequence $E$ corresponding to $e\left(i, a p^{f}\right)$ is $E_{2 * v_{2}^{a p}}$. Take $\bar{\epsilon}_{a p^{f}}=$ $\pi_{r}(\epsilon)^{a p^{f}}: S^{N} W \rightarrow S^{0}$ for suitable $N$, and let $\hat{\epsilon}=\left(\bar{\epsilon}_{a p} f\right) i_{1} ;$ then the diagram

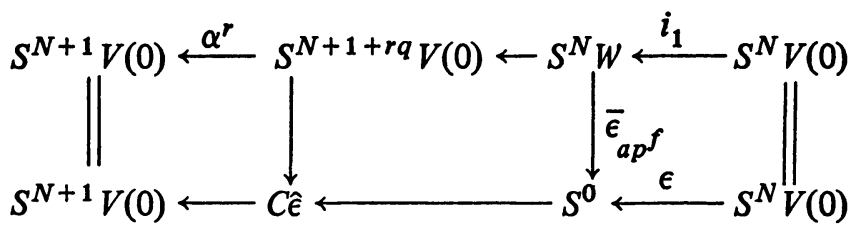

gives rise to a commutative ladder

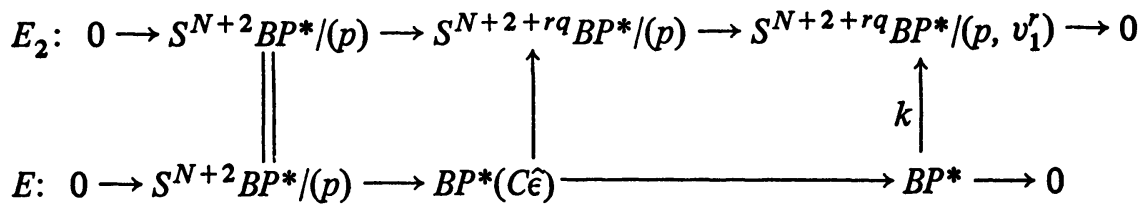

where $k$ is multiplication by $v_{2}^{a p}{ }^{f}$. But this shows that $E$ is the pull-back $E_{2} k$ of $E_{2}$ by $k$, proving the proposition.

Finally, we should show that Proposition 6 and Theorem 1(a) imply that $\epsilon\left(i, a p^{f}\right)$ is essential. First, we can apply the Geometric Boundary Theorem [4] twice, using the Stiefel-Whitney duals of $i$ and $i_{r}$ in place of $h$ in [4], to conclude that $e\left(i, a p^{\dagger}\right)$ is an infinite cycle in the $B P$-homology Adams spectral sequence. But since $e\left(i, a p^{f}\right)$ has filtration 2 , it cannot be a boundary for degree reasons. Therefore it survives to represent a nonzero element of $E_{\infty}$, so in fact $\epsilon\left(i, a p^{f}\right)$ is essential. 
2. Constructing some epsilons. Now that we know how to detect $\epsilon$ 's, we give a simple construction of infinitely many of them in stable homotopy. The method is based on the spectral sequence comparison theorem applied to the $B P$ Adams spectral sequence. In the course of the argument we will find evidence corroborating the fact that $\left(p, v_{1}^{p}, v_{2}^{p}\right)$ is not realizable (cf. [21]).

Let $p \geqslant 5$, and consider the spectral sequence

$$
\operatorname{Ext}_{A}^{s, t}\left(B P^{*}, B P^{*} /\left(p, v_{1}^{r}\right)\right) \Rightarrow[W, S]_{t-s-r q-2}
$$

until further notice we will abbreviate the left-hand side by Ext ${ }^{s, t}$. We know that

$$
\begin{aligned}
\operatorname{Ext}^{0, t} & =\operatorname{Hom}_{A}^{0, t}\left(B P^{*}, B P^{*} /\left(p, v_{1}^{r}\right)\right) \\
& =\left\{y \in B P^{*} /\left(p, v_{1}^{r}\right) \mid\left(p, v_{1}^{r}, y\right) \text { is invariant }\right\}
\end{aligned}
$$

By Lemma 2, then, if $r \leqslant p$, Ext ${ }^{0,\left(p^{2}+p\right) q}$ is isomorphic to $Z / p$, generated by the homomorphism $x$ sending $1 \in B P^{*}$ to $v_{2}^{p}$. If $x$ survives to $E_{\infty}$, it will clearly represent a map $\bar{\epsilon}: W \rightarrow S$ realizing multiplication by $v_{2}^{p}$.

Proposition 8. The element $x$ survives to $E_{\infty}$ if and only if $d_{q+1} x=0$.

Proof. By sparseness and the vanishing theorem [19, 2.1 and 7.1], the only $d_{k} x$ that can have a nonzero range is $d_{q+1}$. For $x$ lies in $\operatorname{Ext}^{0,\left(p^{2}+p\right) q}$, so $d_{k} x \in \operatorname{Ext}^{k,\left(p^{2}+p\right) q+k-1}=0$ unless $k-1 \equiv 0(\bmod q)$ by sparseness. But $d_{l q+1} x \in \operatorname{Ext}^{l q+1},\left(p^{2}+p+l\right) q$, which, by the vanishing theorem, can be a nonzero group only if $\left(p^{2}+p+l\right) q \geqslant(p l(p-1)+1) q$. Since $p$ is odd, this implies $l=1$ as asserted.

It will turn out that $d_{q+1} x$ lies in a nonzero group, so we must perform a closer analysis to show $d_{q+1} x=0$. Let

$$
\begin{gathered}
\cdots \underset{j}{\longrightarrow} \prod_{j} A w_{j}^{(i)} \longrightarrow \cdots \longrightarrow \prod_{j} A w_{j}^{(1)} \\
\stackrel{d_{1}}{\longrightarrow} A \longrightarrow B P^{*} \rightarrow 0
\end{gathered}
$$

by a resolution of $B P^{*}$ by free topologized $A$-modules $[19, \S \S 3,5] . \operatorname{Ext}^{s, t}$ is calculated by applying $\operatorname{Hom}_{A}^{t}\left(-, B P^{*} /\left(p, v_{1}^{\eta}\right)\right)$ to this resolution and taking the cohomology at level s. Actually, it suffices to consider only a locally finite piece of a resolution, which we will call an $(s, T)$-resolution. In $\$ 3$ we will construct an $(s, T)$-resolution adequate for the proof of

THEOREM 10.

$$
\operatorname{Ext}^{q+1,\left(p^{2}+p\right) q-1} \cong\left\{\begin{array}{l}
Z / p \oplus Z / p, \quad r=p \\
Z / p, \quad 1 \leqslant r \leqslant p-1
\end{array}\right.
$$


A generator of the first summand is represented by the cocycle $c: w_{p^{2}}^{(q+1)} \mapsto$ $v_{2}$; if $r=p$, a generator of the other summand is represented by the cocycle $c^{\prime}: w_{p^{2}-p+1}^{(q+1)} \mapsto v_{2} v_{1}^{p-1}$.

Proof of Theorem 1(b). The theorem is true for $r=1$ by the work of Smith [12]. For $1<r<p$, the diagram

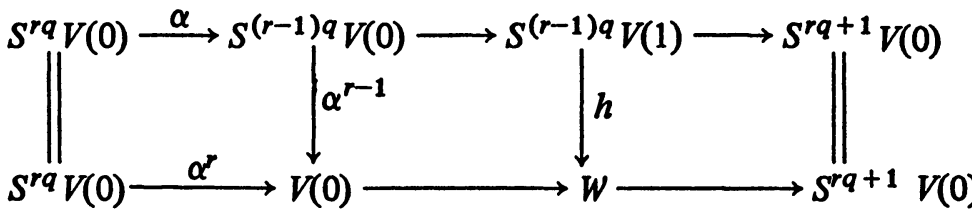

provides a natural map $h: S^{(r-1) q} V(1) \rightarrow W$ inducing the quotient homomorphism $B P^{*} /\left(p, v_{1}^{\eta} \rightarrow B P^{*} /\left(p, v_{1}\right)\right.$. Use $h$ to map the $[W, S]$ spectral sequence to the $[V(1), S]$ sequence. In the latter there is

$$
y \in \operatorname{Ext}^{0,(p+1) q}\left(B P^{*}, B P^{*} /\left(p, v_{1}\right)\right)
$$

represented by the homomorphism sending 1 to $v_{2}$.

There is no element in the $[W, S]$ spectral sequence corresponding to $y$, since $\left(p, v_{1}^{r}, v_{2}\right)$ is not invariant for $r>1$. But $h^{*} x=y^{p}$. Hence

$$
\begin{aligned}
h^{*} d_{q+1} x & =d_{q+1} h^{*} x=d_{q+1} y^{p} \\
& =p y^{p-1} d_{q+1} y \quad \text { (since } y \text { lies in even dimensions) } \\
& =0
\end{aligned}
$$

since $\operatorname{Ext}^{s, t}\left(B P^{*}, B P^{*} /\left(p, v_{1}\right)\right)$ is a $Z / p$-vector space. For degree reasons it follows from Theorem 10 that $d_{q+1} x=k c$ for some $k \in Z / p$. But by naturality and the $r=1$ case, $h^{*}(c) \neq 0$; hence $0=h^{*}\left(d_{q+1} x\right)=k h^{*}(c)$ implies $k=0$.

By Proposition 8 , this proves there is a map $\bar{\epsilon}: W \rightarrow S$ realizing multiplication by $v_{2}^{p}$ in BP-cohomology.

We now assert that for $1 \leqslant r \leqslant p-1$ there is a map $m: W \wedge W \rightarrow W$ extending the identity maps $S^{0} \wedge W \rightarrow W, W \wedge S^{0} \rightarrow W$. This is proved following Toda $\left[17\right.$, p. 59]: it is enough to check that the obstructions in $\pi_{*} W$ to extending $\left(S^{0} \wedge W\right) \vee\left(W \wedge S^{0}\right) \rightarrow W$ to $W \wedge W$ are all zero. The relevant homotopy groups of $W$ are located in gradings $t-s=r q+i, 2 r q+i+1(i=0,1$, 2) of the dual homology spectral sequence to (7). All such $\mathrm{Ext}^{s, t}$ are zero unless $t \neq 0 \bmod q$ and $s=0$, by $\left[19,2.1\right.$ and 7.1]. But $\operatorname{Ext}^{0, *}=B P_{*} /\left(p, v_{1}^{r}\right)$ is zero in these gradings.

Given $m$, take the Spanier-Whitehead dual $D \bar{\epsilon}$; this realizes multiplication by $v_{2}^{p}$ in $B P$-homology. Then it is easy to check that

$$
\epsilon: S^{\left(p^{2}+p\right) q} W \cong S^{\left(p^{2}+p\right) q} \wedge W \stackrel{D \bar{\epsilon} \wedge 1}{\longrightarrow} W \wedge W \stackrel{m}{\longrightarrow} W
$$


also realizes multiplication by $v_{2}^{p}$ in $B P$-homology. Then we can define $\epsilon\left(t, a p^{f}\right)$ by (3). This completes the proof.

Notice that if $r=p, d_{q+1} x$ could well be a nonzero multiple of $c^{\prime}$, since $h^{*} c^{\prime}=0$. Thus the emergence of $c^{\prime}$ is the only obstacle to the realization of the ideal $\left(p, v_{1}^{p}, v_{2}^{p}\right)$. That such a realization is impossible is a corollary of a deep result of Toda (compare [13, Theorem 4.7]).

3. Resolutions revisited. We present now some theorems relating the $A$-resolutions studied in $[19, \S 5]$ to the more classical Ext over the algebra of Steenrod $p$ th powers. This allows us to give a relatively sanitary proof of Theorem 10, obtain a new verification of Miller's theorem on edge periodicity in the $B P$-Adams spectral sequence (generalizing results of $[19, \S 7]$ ), and reinterpret some known results on $V(n)$-spaces. For other new approaches to the calculation of Ext groups over $B P^{*}(B P)$, consult [8] or [2] ; still another method is being investigated by Bendersky [3] .

Recall now the setup of $[19, \S 5]$. There we showed how to compute $\operatorname{Ext}_{A}\left(B P^{*}, B P^{*}\right)$ using locally finite approximations, called $(s, t)$-resolutions, to an $A$-resolution of $B P^{*}$. We need a slight generalization of this notion:

Definition. Let $T=\left(t_{0}, t_{1}, \ldots\right)$ be a sequence of integers, $0 \leqslant t_{0} \leqslant$ $t_{1} \leqslant t_{2} \ldots$. If $s$ is a nonnegative integer, an $(s, T)$-resolution of $B P^{*}$ is a complex

$$
C: C_{s+1} \rightarrow C_{s} \rightarrow \cdots \rightarrow B P^{*} \rightarrow 0
$$

of finite length, consisting of finitely generated free topologized $A$-modules $C_{i}=\bigoplus_{j=1}^{n_{i}} A w_{j}^{(i)}$, such that

$$
\left|w_{j}^{(i)}\right| \leqslant t_{i} \text { for all } i, j
$$

and

$$
\begin{gathered}
\text { for each } i \leqslant s \text { and } t^{\prime} \leqslant t_{i+1}, \\
C\left(i+1, t^{\prime}\right) \rightarrow C\left(i, t^{\prime}\right) \rightarrow C\left(i-1, t^{\prime}\right) \text { is exact over } Z_{(p)} .
\end{gathered}
$$

Then if $C^{\prime}$ is a $\nu$-resolution [19, p. 492], it restricts to an $(s, T)$-resolution $C$ for every $(s, T)$-let $C_{i}=\bigoplus A w_{j}^{(i)}$ where the sum runs over $\left|w_{j}^{(i)}\right| \leqslant t_{i}$. The proof of [19, Proposition 5.2] easily extends to prove that every $(s, T)$-resolution extends to a $\nu$-resolution. Further, [19, Theorem 5.4] holds for $C$ an $\left(s_{0}, T\right)$-resolution if $s \leqslant s_{0}$ and $t \leqslant t_{s}$, allowing us to compute Ext groups. In fact, if $M$ is any finitely generated $A$-module such that $M^{k}=0$ for $k$ larger then some integer $N$, and we let $C^{*}(k, t)=\operatorname{Hom}_{A}^{t}\left(C_{k} / F^{t+1} C_{k}, M\right)$, then Theorem 5.4 holds for the computation of $\operatorname{Ext}_{A}^{s, t}\left(B P^{*}, M\right)$. Our immediate interest, of course, is $M=B P^{*} /\left(p, v_{1}^{r}\right)$. 
Now we want to show that the $A$-generators $w_{j}^{(i)}$ of a suitable $\nu$-resolution correspond exactly to the generators of $\operatorname{Ext}_{p}\left(Z_{p}, Z_{p}\right)$. Recall that if $B P^{*}$ acts on $Z / p \cong B P^{*} /\left(p, v_{1}, v_{2}, \ldots\right)$, we have an isomorphism $A \otimes_{B P^{*}} Z / p \cong P[19$, 3.8].

THEOREM 13 (RESOLUTION LIFTING). Let $\widetilde{C}$ be a minimal resolution of $Z / p$ over $P$. Then there is a v-resolution $C$ of $B P^{*}$ with $C \oplus_{B P *} Z / p \cong \widetilde{C}$.

Proof. Let $\widetilde{C}_{i}=\bigoplus_{j} P \tilde{w}_{j}^{(i)}$, and assume inductively that $C_{i}, d_{i}$ have been constructed for $i \leqslant N$, and $C(N+1, t),\left(d_{N+1}\right)^{t}$ are constructed for $t \leqslant t_{0}$. Pick $\widetilde{w}=\widetilde{w}_{j}^{(N+1)}$ with minimal $|\widetilde{w}|>t_{0} ;$ let $d_{N+1} \widetilde{w}=\Sigma c_{j} P^{E_{j}} \widetilde{w}_{j}^{(N)}, c_{j} \in Z / p$. Then if $x=\Sigma c_{j} r_{E_{j}} w_{j}^{(N)}$ we have inductively that $d_{N} x=p y$ in $C(N-1, t)$, where $t=\left|r_{E_{j}} w_{J}^{(N)}\right|$. But $d_{N-1} p y=0$ in $C(N-2, t)$; hence $d_{N-1} y=0$, so there is $x^{\prime}$ with $d_{N} x^{\prime}=y \bmod F^{t+1}$, since $C$ is an $(N, t)$-resolution. Hence $d_{N}\left(x-p x^{\prime}\right)=0$ in $C(N-1, t)$, so we can introduce $w^{(N+1)}$ with $d_{N+1} w_{j}^{(N+1)}$ $=x-p x^{\prime}$; clearly $d_{N+1} \otimes_{B P^{*}} Z / p=\tilde{d}_{N+1}$. This completes the inductive step and the proof of Theorem 13.

Corollary 14. There is a v-resolution $C$ of $B P^{*}, C_{i}=\bigoplus_{i} A w_{j}^{(i)}$, such that $\bigoplus_{i, j}(Z / p) w_{j}^{(i)}$ is isomorphic as a bigraded $Z / p$-vector space to $\operatorname{Ext}_{p}(Z / p, Z / p)$.

Proof. If $\widetilde{C}$ is a minimal resolution of $Z / p$ over $P, C_{i}=\bigoplus_{j} P w_{j}^{(i)}$, then it is well known that there is one $w_{j}^{(i)}$ in the appropriate dimension for each generator of $\operatorname{Ext}_{p}(Z / p, Z / p)$. The desired $\nu$-resolution is then any lift of $\widetilde{C}$ given by Theorem 13 .

Proposition 15. For $T=\left(t_{1}, t_{2}, \ldots\right)$, where

$$
T_{2 j}=t_{2 j+1}=\left(p^{2}+(j-1) p-1\right) q,
$$

and any $s \geqslant 2$, there is an $(s, T)$-resolution of $B P^{*}$ described as follows:

(16) For $3 \leqslant k \leqslant s+1, C_{k}$ has $A$-generators $w_{i}^{(k)}$ in grading iq for $i=$ $j p, j p+2,(j+1) p+1$ if $k=2 j$, and $i=j p+1,(j+1) p,(j+1) p+2$ if $k$ $=2 j+1$ (if $0 \leqslant k \leqslant 2, C_{k}$ is as in $[19,5.3]$ ).

(17) In terms of this basis, $d_{2 j-1}$ and $d_{2 j}$ are given by the matrices $B$ and $A$ of $[18, \S 7]$, respectively.

REMARK. It is also possible to derive this result by direct calculation over $B P^{*}$.

Proof. By Corollary 14, we may let the elements $w_{i}^{(k)}$ of (16) correspond to the Toda elements [17] $\left(b_{1}^{0}\right)^{j},\left(b_{1}^{0}\right)^{j-1} g_{0},\left(b_{1}^{0}\right)^{j-1} k_{0}$ for $k=2 j$, and $\left(b_{1}^{0}\right)^{j} h_{0}$, $\left(b_{1}^{0}\right)^{j-1} h_{1},\left(b_{1}^{0}\right)^{j-1} h_{0} k_{0}$ for $k=2 j+1$, which form a $Z / p$-basis for $\operatorname{Ext}_{p}(Z / p, Z / p)$ in the indicated gradings. If we then define $d_{k}$ by (17), the 
relations $A B=B A=0$ of [18] and case-by-case checking shows that $\left(C_{k}, d_{k}\right)$ is exact. Hence it is an $(s, T)$-resolution as stated.

Proof of TheOREM 10. Compute $\operatorname{Ext}_{A}^{q+1,\left(p^{2}+p\right) q-1}\left(B P^{*}, B P^{*} /\left(p, v_{1}^{\gamma}\right)\right)$ as a subquotient of $\operatorname{Hom}_{A}^{\left(p^{2}+p+1\right)}\left(C_{q+1}, B P^{* *} /\left(p, v_{1}^{r}\right)\right)$, using the resolution $C$ of Proposition 15. If $p \geqslant 5,1 \leqslant r \leqslant p-1$, the $A$-linear map $c$ defined by

$$
c\left(w f^{(q+1)}\right)= \begin{cases}v_{2}, & j=p^{2}, \\ 0, & \text { otherwise, }\end{cases}
$$

is the only cochain; it is easily seen to be a nontrivial cocycle, since there is also only one $q$-cochain in this grading. (Sample calculation:

hence $d_{q+2}^{*}(c)=0$.)

$$
\begin{aligned}
d_{q+2}^{*}(c)\left(w_{p^{2}}^{(q+2)}\right) & =c\left(d_{q+2} w_{p^{2}}^{(q+2)}\right) \\
& =c\left(\left(-p+v_{1} r_{1}\right) w_{p^{2}}^{(q+1)}+\cdots\right) \\
& =\left(-p+v_{1} r_{1}\right) v_{2} \\
& =0 \bmod \left(p, v_{1}^{r}\right), \text { etc.; }
\end{aligned}
$$

If $r=p$ there are also cochains $w_{p^{2}+2}^{(q+1)} \stackrel{c^{\prime}}{\mapsto} v_{1}^{p-1}$ and $w_{p^{2}-p+1}^{(q+1)} \stackrel{c^{\prime \prime}}{\mapsto}$ $v_{2} v_{1}^{p-1}$ which turn out to be cohomologous cocycles representing another nontrivial Ext element. The details are left to the reader.

Proof of Theorem 1(c). The first half of $1(\mathrm{c})$ is formula (4). Using the BP-Adams spectral sequence or Toda's calculations we can check that $\epsilon(i, p)$ is the only nontrivial element in its stem up to units of $Z / p$, so it must be a unit multiple of Toda's $\epsilon_{i}$.

Finally, as corollaries of the results of this section, we retrieve some results of Miller, Smith, and Toda.

Theorem 18 (Edge Periodicity-cF. [8], [19, §7]). For $T$ as in Proposition $15, s \geqslant 2, t \leqslant t_{s}$,

$$
\operatorname{Ext}_{A}^{s, t}\left(B P^{*}, B P^{*}\right)=\mathrm{Ext}_{A}^{s+2, t+p q}\left(B P^{*}, B P^{*}\right) \text {. }
$$

Proof. This follows at once from Proposition 15 and our observation above that Ext can be computed from an $(s, T)$-resolution. Note that the periodicity operator corresponds to the element $b_{1}^{0}$ of Toda and May.

REMARK. The exact values of these Ext groups are easy to write down, since $\mathrm{Ext}^{2}$ and $\mathrm{Ext}^{3}$ are known in this range.

Theorems 13 and 15 allow us to translate into $B P$ the $Z / p$ calculations of Smith and Toda which lead to proofs of the existence of $V(2)$ (for $p \geqslant 5$ ) and $V(3)$ (for $p \geqslant 7$ ).

Consider the spectral sequence $\operatorname{Ext}_{A}^{s, t}\left(B P^{*}, B P^{*}(V(n))\right) \Rightarrow[V(n), S]$ for $n=1,2$. If we can show that $v_{n+1} \in \mathrm{Ext}^{0}$ survives to $E_{\infty}$, we obtain a map 
$\phi: V(n) \rightarrow S$ realizing multiplication by $v_{n+1}$. For $n=1$ and $p \geqslant 5$ this survival is immediate from the vanishing theorem 7.1 of [19]; for $n=2, p \geqslant$ 7 , the only possible obstruction is in $\operatorname{Ext}^{q+1,\left(p^{2}+p+1\right) q}$. This group is shown to be zero via Proposition 15 and an easy calculation.

We now attempt to extend the map $\phi$ to $V(k)$ for $k \leqslant n$ by induction on n. At each stem the obstructions lie in several Ext groups, which are shown to be all zero by routine calculations based on Proposition 15. The cone of the extended $\phi: V(n) \rightarrow V(n)$ is $V(n+1)$, completing the construction.

\section{REFERENCES}

1. J. F. Adams, On the groups $J(X)$. IV, Topology 5 (1966), 21-71; errata, ibid. 7 (1968), 331. MR 33 \#6628; 37 \#5874.

2; David Baird, private communication.

3. Martin Bendersky, private communication.

4. D. C. Johnson, H. R. Miller, W. S. Wilson and R. S. Zahler, Boundary homomorphisms in the generalized Adams spectral sequence and the nontriviality of infinitely many $\boldsymbol{\gamma}_{t}$ in stable homotopy, Reunión sobre Teoria de Homotopia, edited by Donald Davis, Notas de Matemáticas y Simposia, no. 1, Sociedad Matemáticas Mexicana, Mexico City, 1975, pp. 47-59.

5. D. C. Johnson and R. S. Zahler, Detecting stable homotopy with secondary cobordism operations. II, Rutgers Univ. (preprint).

6. P. S. Landweber, Annihilator ideals and primitive elements in complex cobordism, Illinois J. Math. 17 (1973), 273-284. MR 48 \#1235.

7. J. P. May, Thesis, Princeton Univ.,1964.

8. H. R. Miller, Thesis, Princeton Univ., 1975.

9. H. R. Miller and W. S. Wilson, On Novikov's Ext ${ }^{1}$ modulo an invariant prime ideal, Topology 15 (1976), $131-141$.

10. S. P. Novikov, Methods of algebraic topology from the viewpoint of cobordism theories, Izv. Akad. Nauk SSSR Ser. Mat. 1 (1967), 855-951 = Math. USSR Izv. 1 (1967) 827-922. MR 36 \#4561.

11. S. Oka, A new family in the stable homotopy groups of spheres, Hiroshima Math. J. 5 (1975), 87-114.

12. L. Smith, On realizing complex bordism modules. I. Applications to the stable homotopy of spheres, Amer. J. Math. 92 (1970), 793-856. MR 43 \#1186a.

13. - On realizing complex bordism modules. II. Applications to the stable homotopy group of spheres, Amer. J. Math. 93 (1971), 226-263. MR 43 \#1186b.

14. - On realizing complex bordism modules. IV, Indiana Univ. (preprint).

15. M. C. Tangora, private communcation.

16. H. Toda, p-primary components of homotopy groups. IV, Mem. Coll. Sci. Univ.

Kyoto Ser. A Math. 32 (1959), 297-332. MR 22 \#1906.

17. - On spectra realizing exterior parts of the Steenrod algebra, Topology 10 (1971), 53-65. MR 42 \#6814.

18. P. E. Thomas and R. S. Zahler, Generalized higher order cohomology operations and stable homotopy groups of spheres, Advances in Math. 20 (1976), 289-328.

19. R. S. Zahler, The Adams-Novikov spectral sequence for the spheres, Ann. of Math. (2) 96 (1972), 480-504. MR 47 \#7742.

20. - Detecting stable homotopy with secondary cobordism operations. I, Quart. J. Math. Oxford Ser. (2) 25 (1974), 213-226. MR 51 \#4240.

21. Nonrealizability of some cyclic complex bordism modules, Proc. Amer. Math. Soc. 47 (1975), 218-222. MR 50 \#14748.

DEPARTMENT OF MATHEMATICS, DOUGLASS COLLEGE, RUTGERS UNIVERSITY, NEW BRUNSWICK, NEW JERSEY 08903

Current address: 104 Hubinger Street, New Haven, Connecticut 06511 